\title{
Answer to Medical Quiz: Images
}

(BIRDEM Med J 2019; 9(1): 81)

1. X-ray lumbo-sacral spine shows severe osteopenia and compression collapse of T12 and L1 vertebrae.

$\mathrm{X}$-ray skull lateral view shows multiple small punched out osteolytic lesions of different sizes in the skull.

2. Multiple myeloma

3. Serum protein electrophoresis

Urine for Bence Jones protein

Bone marrow examination

\section{Review}

Multiple myeloma is the malignant proliferation of plasma cells producing abnormal monoclonal immunoglobulin or its fragment (M protein). Although a small number of malignant plasma cells are present in the circulation, the majority are present in the bonemarrow. The malignant plasma cells produce cytokines, which stimulate osteoclast causing bone resorption.

Diagnostic criteria ${ }^{1}$ includes any 2 of the following:

1. Increased malignant plasma cells in bone marrow

2. Serum and/or urinary M protein

3. Skeletal lytic lesions

Causes of renal damage in multiple myeloma:

Renal involvement occurs in $20-40 \%$ of newly diagnosed multiple myeloma patients. Causes are:

1. Cast nephropathy - most common (60 - 70\%)

2. Light chain deposition disease (20 - 40\%)
3. Amyloidosis

4. Others: urate nephropathy, hypercalcaemia, drugs etc.

\section{Treatment}

Treatment of offending factors: dehydration, infection, hypercalcaemia, hyperuricaemia

Anti-myeloma agents: chemotherapy (bortezomib and dexamethasone based), autologous stem cell transplantation

Prognostic factors:

Beta-2 microglobulin

Serum albumin

Serum lactate dehydrogenase (LDH) level

C-reactive protein (CRP)

Renal failure

Advanced lytic lesions

Low haemoglobin

Age $>65$ years

Bone marrow cytogenetics and fluorescence in situ hybridization (FISH)

\section{References}

1. Watson HG, Culligan DJ, Manson LM, Haematology and Trnsfusion Medicine. In: Ralston SH, Perman ID, Strachan MWJ, Hobson RP (Eds). Davidson's Principle and Practice of Medicine. 23rd edition. Edingurgh. ELSEVIER 2018; pp966-968. 


\section{Answer to Medical Quiz: SBA}

(BIRDEM Med J 2019; 9(1): 82)

\section{Question No. 1: Correct Answer - B}

The description of bone pain and bowing of an extremity are highly suggestive of Paget's disease of the bone and the increased heat is secondary to a high vascular supply to the affected region. In addition to these symptoms, Paget's can also affect the skull, leading to frontal bossing, headaches, hearing loss and trapped nerves. Paget's disease is a consequence of excessive osteoclastic bone resorption followed by disorganized and uncoordinated osteoblastic bone formation, which leads to weakened and deformed bones. Patients with Paget's disease have a $1 \%$ chance of going on to develop sarcoma.

\section{Question No. 2: Correct Answer - C}

This patient has acute on chronic renal failure, as evidenced by the deteriorated creatinine level, which has led to the accumulation of renally excreted drugs such as digoxin and resulted in digoxin toxicity. This is manifested by nausea and vomiting, which might underlie her renal deterioration but which are also nonspecific symptoms of digoxin toxicity. More characteristically, she describes xanthopsia, or a yellow tinge to lights and objects. Cardiac manifestations of excess digoxin are bradyarrhythmias, ventricular ectopics, variable degrees of heart block and, rarely, ventricular tachycardia or fibrillation. In patients with digoxin toxicity it is vital to measure potassium levels as hypokalaemia potentiates the cardiac effects of digoxin and even patients with therapeutic digoxin levels can develop toxicity in the context of hypokalaemia.

\section{Question No. 3: Correct Answer - A}

This patient presents with the triad of weight loss, abdominal pain and diarrhoea, which commonly occurs in Crohn's disease. This is supported by his constitutional symptoms and anal skin tags. Rose-thorn ulceration is seen on barium studies and not at sigmoidoscopy. Transmural granulomatous inflammation occurs in Crohn's whereas superficial mucosal ulceration occurs in ulcerative colitis. As inflammation is transmural it allows fistula formation between adjacent anatomical structures such as bowel and skin (enterocutaneous) and bowel and bladder (colovesical). Both ulcerative colitis and Crohn's disease can be complicated by osteomalacia and by toxic dilatation of the colon, which is a surgical emergency.

\section{Question No. 4: Correct Answer - B}

The main differential in this patient is hyperaldosteronism arising from an adrenal tumour (Conn's syndrome). The excess aldosterone causes hypertension, elevated sodium reabsorption and potassium excretion. However, given the patient history, the elevated blood pressure could easily be due to the stress of having blood pressure measured or the patient's personal situation. A 24-hour ambulatory blood pressure measurement is therefore the most appropriate investigation to eliminate essential hypertension. Since the blood results are only mildly deranged and essential hypertension has not been eliminated, an aldosteronerenin ratio, CT scan or abdominal ultrasound would not be the first-line investigations to consider. They would be useful to investigate Conn's syndrome if essential hypertension was excluded as a differential. A glucose tolerance test is inappropriate in this case since the random glucose reading is not abnormal and the patient is not suffering from symptoms suggestive of diabetes.

\section{Question No. 5: Correct Answer - A}

Toxoplasmosis is caused by the protozoa Toxoplasmosis gondii. In patients with HIV, it may present with encephalitis. Clinical features include fever, headache confusion and convulsions. As in this case, there may be focal neurological signs on examination. The multiple ring enhancing lesions are the characteristic $\mathrm{CT}$ finding. Meningitis may present with a high fever, headache and confusion. However, the focal neurological signs and the presence of multiple ring-enhancing lesions on CT are unlikely. Cryptosporidiosis may affect HIV patients and presents with abdominal pain, nausea and vomiting and profuse diarrhoea. While CMV encephalitis may produce the clinical features described in this question, the CT finding of the multiple ring enhancing lesions makes this answer incorrect. Histoplasmosis may affect HIV patients and presents with respiratory symptoms. 